\title{
A $q$-analogue of some binomial coefficient identities of Y. Sun
}

\author{
Victor J. W. Guo ${ }^{1}$ and Dan-Mei Yang ${ }^{2}$ \\ Department of Mathematics, East China Normal University \\ Shanghai 200062, People's Republic of China \\ ${ }^{1}$ jwguo@math.ecnu.edu.cn, ${ }^{2}$ plain_dan2004@126.com
}

Submitted: Dec 1, 2010; Accepted: Mar 24, 2011; Published: Mar 31, 2011

Mathematics Subject Classifications: 05A10, 05A17

\begin{abstract}
We give a $q$-analogue of some binomial coefficient identities of Y. Sun [Electron. J. Combin. 17 (2010), \#N20] as follows:

$$
\sum_{k=0}^{\lfloor n / 2\rfloor}\left[\begin{array}{c}
m+k \\
k
\end{array}\right]_{q^{2}}\left[\begin{array}{c}
m+1 \\
n-2 k
\end{array}\right]_{q} q^{\left(\begin{array}{c}
n-2 k \\
2
\end{array}\right)}=\left[\begin{array}{c}
m+n \\
n
\end{array}\right]_{q}
$$$$
\sum_{k=0}^{\lfloor n / 4\rfloor}\left[\begin{array}{c}
m+k \\
k
\end{array}\right]_{q^{4}}\left[\begin{array}{c}
m+1 \\
n-4 k
\end{array}\right]_{q} q^{\left(\begin{array}{c}
n-4 k \\
2
\end{array}\right)}=\sum_{k=0}^{\lfloor n / 2\rfloor}(-1)^{k}\left[\begin{array}{c}
m+k \\
k
\end{array}\right]_{q^{2}}\left[\begin{array}{c}
m+n-2 k \\
n-2 k
\end{array}\right]_{q},
$$

where $\left[\begin{array}{l}n \\ k\end{array}\right]_{q}$ stands for the $q$-binomial coefficient. We provide two proofs, one of which is combinatorial via partitions.

\section{Introduction}

Using the Lagrange inversion formula, Mansour and Sun [2] obtained the following two binomial coefficient identities:

$$
\begin{aligned}
\sum_{k=0}^{\lfloor n / 2\rfloor} \frac{1}{2 k+1}\left(\begin{array}{c}
3 k \\
k
\end{array}\right)\left(\begin{array}{c}
n+k \\
3 k
\end{array}\right) & =\frac{1}{n+1}\left(\begin{array}{c}
2 n \\
n
\end{array}\right), \\
\sum_{k=0}^{\lfloor(n-1) / 2\rfloor} \frac{1}{2 k+1}\left(\begin{array}{c}
3 k+1 \\
k+1
\end{array}\right)\left(\begin{array}{c}
n+k \\
3 k+1
\end{array}\right) & =\frac{1}{n+1}\left(\begin{array}{c}
2 n \\
n
\end{array}\right) \quad(n \geqslant 1) .
\end{aligned}
$$


In the same way, Sun [3] derived the following binomial coefficient identities

$$
\begin{aligned}
\sum_{k=0}^{\lfloor n / 2\rfloor} \frac{1}{3 k+a}\left(\begin{array}{c}
3 k+a \\
k
\end{array}\right)\left(\begin{array}{c}
n+a+k-1 \\
n-2 k
\end{array}\right) & =\frac{1}{2 n+a}\left(\begin{array}{c}
2 n+a \\
n
\end{array}\right), \\
\sum_{k=0}^{\lfloor n / 4\rfloor} \frac{1}{4 k+1}\left(\begin{array}{c}
5 k \\
k
\end{array}\right)\left(\begin{array}{c}
n+k \\
5 k
\end{array}\right) & =\sum_{k=0}^{\lfloor n / 2\rfloor} \frac{(-1)^{k}}{n+1}\left(\begin{array}{c}
n+k \\
k
\end{array}\right)\left(\begin{array}{c}
2 n-2 k \\
n
\end{array}\right), \\
\sum_{k=0}^{\lfloor n / 4\rfloor} \frac{n+a+1}{4 k+a+1}\left(\begin{array}{c}
5 k+a \\
k
\end{array}\right)\left(\begin{array}{c}
n+a+k \\
5 k+a
\end{array}\right) & =\sum_{k=0}^{\lfloor n / 2\rfloor}(-1)^{k}\left(\begin{array}{c}
n+a+k \\
k
\end{array}\right)\left(\begin{array}{c}
2 n+a-2 k \\
n+a
\end{array}\right) .
\end{aligned}
$$

It is not hard to see that both (1.1) and (1.2) are special cases of (1.3), and (1.4) is the $a=0$ case of (1.5). A bijective proof of (1.1) and (1.3) using binary trees and colored ternary trees has been given by Sun [3] himself. Using the same model, Yan [4] presented an involutive proof of (1.4) and (1.5), answering a question of Sun.

Multiplying both sides of (1.3) by $n+a$ and letting $m=n+a-1$, we may write it as

$$
\sum_{k=0}^{\lfloor n / 2\rfloor}\left(\begin{array}{c}
m+k \\
k
\end{array}\right)\left(\begin{array}{c}
m+1 \\
n-2 k
\end{array}\right)=\left(\begin{array}{c}
m+n \\
n
\end{array}\right),
$$

while letting $m=n+a$, we may write (1.5) as

$$
\sum_{k=0}^{\lfloor n / 4\rfloor}\left(\begin{array}{c}
m+k \\
k
\end{array}\right)\left(\begin{array}{c}
m+1 \\
n-4 k
\end{array}\right)=\sum_{k=0}^{\lfloor n / 2\rfloor}(-1)^{k}\left(\begin{array}{c}
m+k \\
k
\end{array}\right)\left(\begin{array}{c}
m+n-2 k \\
m
\end{array}\right) .
$$

The purpose of this paper is to give a $q$-analogue of (1.6) and (1.7) as follows:

$$
\begin{aligned}
& \sum_{k=0}^{\lfloor n / 2\rfloor}\left[\begin{array}{c}
m+k \\
k
\end{array}\right]_{q^{2}}\left[\begin{array}{c}
m+1 \\
n-2 k
\end{array}\right]_{q} q^{\left(\begin{array}{c}
n-2 k \\
2
\end{array}\right)}=\left[\begin{array}{c}
m+n \\
n
\end{array}\right]_{q}, \\
& \sum_{k=0}^{\lfloor n / 4\rfloor}\left[\begin{array}{c}
m+k \\
k
\end{array}\right]_{q^{4}}\left[\begin{array}{c}
m+1 \\
n-4 k
\end{array}\right]_{q} q^{\left(\begin{array}{c}
n-4 k \\
2
\end{array}\right)}=\sum_{k=0}^{\lfloor n / 2\rfloor}(-1)^{k}\left[\begin{array}{c}
m+k \\
k
\end{array}\right]_{q^{2}}\left[\begin{array}{c}
m+n-2 k \\
n-2 k
\end{array}\right]_{q},
\end{aligned}
$$

where the $q$-binomial coefficient $\left[\begin{array}{l}x \\ k\end{array}\right]_{q}$ is defined by

$$
\left[\begin{array}{l}
x \\
k
\end{array}\right]_{q}= \begin{cases}\prod_{i=1}^{k} \frac{1-q^{x-i+1}}{1-q^{i}}, & \text { if } k \geqslant 0 \\
0, & \text { if } k<0 .\end{cases}
$$

We shall give two proofs of (1.8) and (1.9). One is combinatorial and the other algebraic. 


\section{Bijective proof of (1.8)}

Recall that a partition $\lambda$ is defined as a finite sequence of nonnegative integers $\left(\lambda_{1}, \lambda_{2}\right.$, $\left.\ldots, \lambda_{r}\right)$ in decreasing order $\lambda_{1} \geqslant \lambda_{2} \geqslant \cdots \geqslant \lambda_{r}$. A nonzero $\lambda_{i}$ is called a part of $\lambda$. The number of parts of $\lambda$, denoted by $\ell(\lambda)$, is called the length of $\lambda$. Write $|\lambda|=\sum_{i=1}^{m} \lambda_{i}$, called the weight of $\lambda$. The sets of all partitions and partitions into distinct parts are denoted by $\mathscr{P}$ and $\mathscr{D}$ respectively. For two partitions $\lambda$ and $\mu$, let $\lambda \cup \mu$ be the partition obtained by putting all parts of $\lambda$ and $\mu$ together in decreasing order.

It is well known that (see, for example, [1, Theorem 3.1])

$$
\begin{aligned}
& \sum_{\substack{\lambda_{1} \leqslant m+1 \\
\ell(\lambda)=n}} q^{|\lambda|}=q^{n}\left[\begin{array}{c}
m+n \\
n
\end{array}\right]_{q}, \\
& \sum_{\substack{\lambda \in \mathscr{D} \\
\lambda \leqslant 1 \\
\ell(\lambda)=n}} q^{|\lambda|}=\left[\begin{array}{c}
m+1 \\
n
\end{array}\right]_{q} q^{\left(\begin{array}{c}
n+1 \\
2
\end{array}\right) .}
\end{aligned}
$$

Therefore,

$$
\sum_{\substack{\mu \in \mathscr{D} \\
\lambda_{1}, \mu_{1} \leqslant m+1 \\
2 \ell(\lambda)+\ell(\mu)=n}} q^{2|\lambda|+|\mu|}=q^{n} \sum_{k=0}^{\lfloor n / 2\rfloor}\left[\begin{array}{c}
m+k \\
k
\end{array}\right]_{q^{2}}\left[\begin{array}{c}
m+1 \\
n-2 k
\end{array}\right]_{q} q^{\left(\begin{array}{c}
n-2 k \\
2
\end{array}\right)},
$$

where $k=\ell(\lambda)$. Let

$$
\begin{aligned}
& \mathscr{A}=\left\{\lambda \in \mathscr{P}: \lambda_{1} \leqslant m+1 \text { and } \ell(\lambda)=n\right\}, \\
& \mathscr{B}=\left\{(\lambda, \mu) \in \mathscr{P} \times \mathscr{D}: \lambda_{1}, \mu_{1} \leqslant m+1 \text { and } 2 \ell(\lambda)+\ell(\mu)=n\right\} .
\end{aligned}
$$

We shall construct a weight-preserving bijection $\phi$ from $\mathscr{A}$ to $\mathscr{B}$. For any $\lambda \in \mathscr{A}$, we associate it with a pair $(\bar{\lambda}, \mu)$ as follows: If $\lambda_{i}$ appears $r$ times in $\lambda$, then we let $\lambda_{i}$ appear $\lfloor r / 2\rfloor$ times in $\bar{\lambda}$ and $r-2\lfloor r / 2\rfloor$ times in $\mu$. For example, if $\lambda=(7,5,5,4,4,4,4,2,2,2,1)$, then $\bar{\lambda}=(5,4,4,2)$ and $\mu=(7,2,1)$. Clearly, $(\bar{\lambda}, \mu) \in \mathscr{B}$ and $|\lambda|=2|\bar{\lambda}|+|\mu|$. It is easy to see that $\phi: \lambda \mapsto(\bar{\lambda}, \mu)$ is a bijection. This proves that

$$
\sum_{\lambda \in \mathscr{A}} q^{|\lambda|}=\sum_{(\lambda, \mu) \in \mathscr{B}} q^{2|\lambda|+|\mu|} .
$$

Namely, the identity (1.8) holds. 


\section{Involutive proof of (1.9)}

It is easy to see that

$$
\begin{aligned}
q^{n} \sum_{k=0}^{\lfloor n / 2\rfloor}(-1)^{k}\left[\begin{array}{c}
m+k \\
k
\end{array}\right]_{q^{2}}\left[\begin{array}{c}
m+n-2 k \\
n-2 k
\end{array}\right]_{q} & =\sum_{k=0}^{\lfloor n / 2\rfloor}(-1)^{k} \sum_{\substack{\lambda_{1} \leqslant m+1 \\
\ell(\lambda)=k}} q^{2|\lambda|} \sum_{\substack{\mu_{1} \leqslant m+1 \\
\ell(\mu)=n-2 k}} q^{|\mu|} \\
& =\sum_{\substack{\lambda_{1}, \mu_{1} \leqslant m+1 \\
2 \ell(\lambda)+\ell(\mu)=n}}(-1)^{\ell(\lambda)} q^{2|\lambda|+|\mu|},
\end{aligned}
$$

and

$$
q^{n} \sum_{k=0}^{\lfloor n / 4\rfloor}\left[\begin{array}{c}
m+k \\
k
\end{array}\right]_{q^{4}}\left[\begin{array}{c}
m+1 \\
n-4 k
\end{array}\right]_{q} q^{\left(\frac{n-4 k}{2}\right)}=\sum_{\substack{\mu \in \mathscr{D} \\
\lambda, \mu 1 \\
\lambda_{1} \leqslant m+1 \\
\ell(\lambda)+\ell(\mu)=n}} q^{4|\lambda|+|\mu|} .
$$

Let

$$
\begin{array}{r}
\mathscr{U}=\left\{(\lambda, \mu) \in \mathscr{P} \times \mathscr{P}: \lambda_{1}, \mu_{1} \leqslant m+1 \text { and } 2 \ell(\lambda)+\ell(\mu)=n\right\}, \\
\mathscr{V}=\left\{(\lambda, \mu) \in \mathscr{U}: \text { each } \lambda_{i} \text { appears an even number of times and } \mu \in \mathscr{D}\right\} .
\end{array}
$$

We shall construct an involution $\theta$ on the set $\mathscr{U} \backslash \mathscr{V}$ with the properties that $\theta$ preserves $2|\lambda|+|\mu|$ and reverses the sign $(-1)^{\ell(\lambda)}$.

For any $(\lambda, \mu) \in \mathscr{U} \backslash \mathscr{V}$, notice that either some $\lambda_{i}$ appears an odd number of times in $\lambda$, or some $\mu_{j}$ is repeated in $\mu$, or both are true. Choose the largest such $\lambda_{i}$ and $\mu_{j}$ if they exist, denoted by $\lambda_{i_{0}}$ and $\mu_{j_{0}}$ respectively. Define

$$
\theta((\lambda, \mu))= \begin{cases}\left(\left(\lambda \backslash \lambda_{i_{0}}\right), \mu \cup\left(\lambda_{i_{0}}, \lambda_{i_{0}}\right)\right), & \text { if } \lambda_{i_{0}} \geqslant \mu_{j_{0}} \text { or } \mu \in \mathscr{D}, \\ \left(\left(\lambda \cup \mu_{j_{0}}\right), \mu \backslash\left(\mu_{j_{0}}, \mu_{j_{0}}\right)\right), & \text { if } \lambda_{i_{0}}<\mu_{j_{0}} \text { or } \lambda_{i_{0}} \text { does not exist. }\end{cases}
$$

For example, if $\lambda=(5,5,4,4,4,3,3,3,1,1)$ and $\mu=(5,3,2,2,1)$, then

$$
\theta(\lambda, \mu)=((5,5,4,4,3,3,3,1,1),(5,4,4,3,2,2,1)) .
$$

It is easy to see that $\theta$ is an involution on $\mathscr{U} \backslash \mathscr{V}$ with the desired properties. This proves that

$$
\begin{aligned}
\sum_{(\lambda, \mu) \in \mathscr{U}}(-1)^{\ell(\lambda)} q^{2|\lambda|+|\mu|} & =\sum_{\substack{(\lambda, \mu) \in \mathscr{V}\\
}}(-1)^{\ell(\lambda)} q^{2|\lambda|+|\mu|} \\
& \sum_{\substack{\mu \in \mathscr{D} \\
\tau_{1}, \mu_{1} \leqslant m+1 \\
4 \ell(\tau)+\ell(\mu)=n}} q^{4|\tau|+|\mu|}
\end{aligned}
$$

where $\lambda=\tau \cup \tau$. Combining (3.1)-(3.3), we complete the proof of (1.9). 


\section{Generating function proof of (1.8) and (1.9)}

Recall that the q-shifted factorial is defined by

$$
(a ; q)_{0}=1, \quad(a ; q)_{n}=\prod_{k=0}^{n-1}\left(1-a q^{k}\right), n=1,2, \ldots
$$

Then we have

$$
\begin{aligned}
& \frac{1}{\left(z^{2} ; q^{2}\right)_{m+1}}(-z ; q)_{m+1}=\frac{1}{(z ; q)_{m+1}} \\
& \frac{1}{\left(z^{4} ; q^{4}\right)_{m+1}}(-z ; q)_{m+1}=\frac{1}{(z ; q)_{m+1}} \frac{1}{\left(-z^{2} ; q^{2}\right)_{m+1}}
\end{aligned}
$$

By the $q$-binomial theorem (see, for example, [1, Theorem 3.3]), we may expand (4.1) and (4.2) respectively as follows:

$$
\begin{aligned}
& \left(\sum_{k=0}^{\infty}\left[\begin{array}{c}
m+k \\
k
\end{array}\right]_{q^{2}} z^{2 k}\right)\left(\sum_{k=0}^{m+1}\left[\begin{array}{c}
m+1 \\
k
\end{array}\right]_{q} q^{\left(\begin{array}{l}
k \\
2
\end{array}\right) z^{k}}\right)=\sum_{k=0}^{\infty}\left[\begin{array}{c}
m+k \\
k
\end{array}\right]_{q} z^{k}, \\
& \left(\sum_{k=0}^{\infty}\left[\begin{array}{c}
m+k \\
k
\end{array}\right]_{q^{4}} z^{4 k}\right)\left(\sum_{k=0}^{m+1}\left[\begin{array}{c}
m+1 \\
k
\end{array}\right]_{q} q^{\left(\begin{array}{c}
k \\
2
\end{array}\right) z^{k}}\right) \\
& =\left(\sum_{k=0}^{\infty}\left[\begin{array}{c}
m+k \\
k
\end{array}\right]_{q} z^{k}\right)\left(\sum_{k=0}^{\infty}\left[\begin{array}{c}
m+k \\
k
\end{array}\right]_{q^{2}}(-1)^{k} z^{2 k}\right) .
\end{aligned}
$$

Comparing the coefficients of $z^{n}$ in both sides of (4.3) and (4.4), we obtain (1.8) and (1.9) respectively.

Finally, we give the following special cases of (1.8):

$$
\begin{gathered}
\sum_{k=0}^{\lfloor n / 2\rfloor}\left[\begin{array}{c}
n+k \\
k
\end{array}\right]_{q^{2}}\left[\begin{array}{c}
n+1 \\
2 k+1
\end{array}\right]_{q} q^{\left(\begin{array}{c}
n-2 k \\
2
\end{array}\right)}=\left[\begin{array}{c}
2 n \\
n
\end{array}\right]_{q}, \\
\sum_{k=0}^{\lfloor n / 2\rfloor}\left[\begin{array}{l}
n+k \\
k+1
\end{array}\right]_{q^{2}}\left[\begin{array}{c}
n \\
2 k+1
\end{array}\right]_{q} q^{\left(\begin{array}{c}
n-2 k-1 \\
2
\end{array}\right)}=\left[\begin{array}{c}
2 n \\
n-1
\end{array}\right]_{q} .
\end{gathered}
$$

When $q=1$, the identities (4.5) and (4.6) reduce to (1.1) and (1.2) respectively.

Acknowledgments. This work was partially supported by the Fundamental Research Funds for the Central Universities, Shanghai Rising-Star Program (\#09QA1401700), Shanghai Leading Academic Discipline Project (\#B407), and the National Science Foundation of China (\#10801054). 


\section{References}

[1] G. E. Andrews, The Theory of Partitions, Cambridge University Press, Cambridge, 1998.

[2] T. Mansour and Y. Sun, Bell polynomials and $k$-generalized Dyck paths, Discrete Appl. Math. 156 (2008), 2279-2292.

[3] Y. Sun, A simple bijection between binary trees and colored ternary trees, Electron. J. Combin. 17 (2010), \#N20.

[4] S. H. F. Yan, Bijective proofs of identities from colored binary trees, Electron. J. Combin. 15 (2008), \#N20. 